Provided for non-commercial research and education use. Not for reproduction, distribution or commercial use.

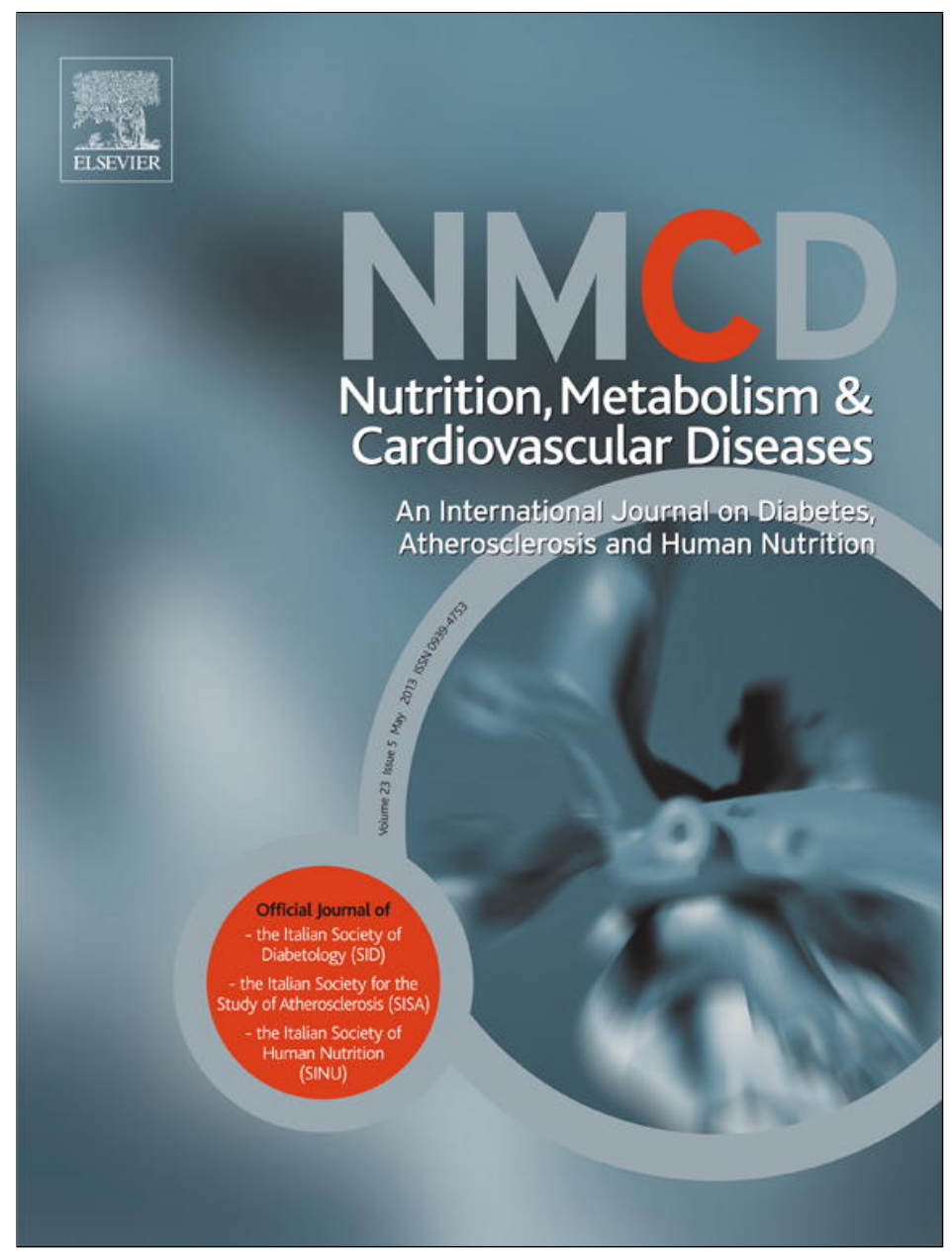

This article appeared in a journal published by Elsevier. The attached copy is furnished to the author for internal non-commercial research and education use, including for instruction at the authors institution and sharing with colleagues.

Other uses, including reproduction and distribution, or selling or licensing copies, or posting to personal, institutional or third party websites are prohibited.

In most cases authors are permitted to post their version of the article (e.g. in Word or Tex form) to their personal website or institutional repository. Authors requiring further information regarding Elsevier's archiving and manuscript policies are encouraged to visit:

http://www.elsevier.com/authorsrights 


\title{
Similar prediction of total mortality, diabetes incidence and cardiovascular events using relative- and absolute-component Mediterranean diet score: The SUN cohort
}

\author{
L.J. Domínguez ${ }^{a}, M$. Bes-Rastrollo ${ }^{b}, C$. de la Fuente-Arrillaga ${ }^{b}, E$. Toledo ${ }^{b}$, \\ J.J. Beunza ${ }^{b}$, M. Barbagallo ${ }^{a, *}$, M.A. Martínez-González ${ }^{b}$
}

\author{
a Geriatric Unit, Department of Internal Medicine and Geriatrics, University of Palermo, via F. Scaduto 6/c, \\ 90144 Palermo, Italy \\ ${ }^{\mathrm{b}}$ Department of Preventive Medicine and Public Health, Medical School, Clinica Universidad de Navarra, \\ University of Navarra, Spain
}

Received 20 March 2011; received in revised form 30 September 2011; accepted 18 October 2011 Available online 7 March 2012

\section{KEYWORDS \\ Mediterranean diet; \\ Cardiovascular \\ disease; \\ Diabetes; \\ Macronutrients}

\begin{abstract}
Background and Aim: Accumulated evidence supports the effectiveness of Mediterranean-type diets (MeDiet) in reducing mortality and preventing several chronic diseases. Widely used scores to assess adherence to MeDiet are based on specific sample characteristics; alternatively, they might be built according to absolute/normative cut-off points for the consumption of specific food groups (pre-defined servings/day or/week). The aim of this study was to compare sample-specific MeDiet adherence scores (MDS) versus absolutenormative scores (Mediterranean Diet Adherence Screener - MEDAS) on their association with macronutrient intake, total mortality and incidence of chronic diseases. Design: SUN (Seguimiento Universidad de Navarra) dynamic prospective cohort study (60.5\% women; mean age 38.4 years).

Methods and Results: In cross-sectional analyses $(n=20,155)$ we evaluated macronutrient distribution according to MDS (based on 136-item FFQ), MEDAS (based on 13 questions), and variants of both. In prospective analyses ( $n=9109$; mean follow-up: 6.2 years), we evaluated disease incidence or mortality. Adherence to MeDiet increased with age and, as expected, was associated with higher fiber intake, lower total fat intake but higher monounsaturated/saturated fat ratio, using all scores. Among subjects initially free of cancer, diabetes, and cardiovascular disease (CVD), adherence to MeDiet appraised with an absolute-normative score (MEDAS) similarly predicted macronutrient distribution and disease incidence or mortality
\end{abstract}

\footnotetext{
* Corresponding author. Tel.: +39091 6552885; fax: +390916552952.

E-mail address: mabar@unipa.it (M. Barbagallo).
} 
(diabetes incidence, CVD or all-cause mortality), when compared to a sample-specific score based on 136-item FFQ (MDS).

Conclusions: Adherence to MeDiet was associated with a decreased incidence of a composite outcome including diabetes incidence, cardiovascular events incidence or all-cause mortality. (c) 2011 Elsevier B.V. All rights reserved.

\section{Introduction}

The traditional Mediterranean diet (MeDiet) has compelling evidence of positive effects [1], comprising reductions in mortality [2,3], incidence of Alzheimer's disease, Parkinson's disease [1], type 2 diabetes [4], cardiovascular events [5,6], stroke [5], and depression [7]. Even if most of this evidence comes from observational studies, a recent intervention RCT (Prevención con Dieta Mediterránea PREDIMED) has shown that MeDiet is able to prevent the development of diabetes in subjects with high cardiovascular risk [8]. To appraise adherence to MeDiet, scores attributing positive points to traditional components of this dietary pattern thought to be protective against adverse health outcomes, and negative points to foods thought to be associated with unfavorable outcomes, have been extensively used [9] since their introduction by Trichopoulou et al. [10], and have been consistently confirmed in Mediterranean [2,4] and non-Mediterranean populations $[3,11]$. However, they are highly dependent on the sample characteristics because they use sex-specific medians of consumption for that sample to define the cut-off points classifying the item consumption as high or low. This makes comparisons among different populations/studies and individual risk assessment unfeasible.

An alternative is to use pre-defined absolute or normative goals of consumption for specific food items, independent of the sample characteristics, such as the questionnaire used in the PREDIMED study [12-14]. This questionnaire can be used by personnel without highly specialized training or can be self-administered, enlarging the possibility of a real-time estimation of risk.

Most of the available scores assessing adherence to MeDiet allocate the same weight to its components. However, different components can have different relationships with health outcomes, as reported by a recent meta-analysis of prospective cohort studies and RCTs for coronary heart disease (CHD) [15]. Moderate ethanol intake, low intake of meat and high consumption of vegetables appear to drive the association of adherence to MeDiet with lower mortality [16].

The present study aimed: 1) to evaluate, in crosssectional analyses, the macronutrient distribution according to adherence to MeDiet assessed with both, a samplespecific (MDS) or an absolute-normative (Mediterranean Diet Adherence Screener - MEDAS) scoring system, considering also the effect of adjusting each item for total energy intake or of weighting each item by its evidence-based contribution to CHD protection; 2 ) to evaluate, in a longitudinal analysis, the association of adherence to MeDiet (MDS or MEDAS scores) with a composite outcome of cardiovascular events incidence, type 2 diabetes incidence or all-cause mortality, in the SUN - Seguimiento Universidad de Navarra - project.

\section{Methods}

\section{Study population}

The SUN is a prospective, permanently open, dynamic cohort of university graduates started in 1999. The design and methods of the SUN study have been previously described in detail [17]. The study protocol was approved by the Institutional Review Board of the University of Navarra and the initial response to the mailed questionnaire was considered as informed consent to participate. For the present analyses we used the database up to September 2009 ( $n=20,349$ participants). For the evaluation of adherence to MeDiet according to MDS and MEDAS, the whole population was considered, excluding participants with energy intake out of pre-defined limits (men: $<800$ or $>8000 \mathrm{kcal} /$ day; women: $<500$ or $>6000 \mathrm{kcal} / \mathrm{day})(n=20,155$ participants $)$ in order to capture extreme values of energy in the cross-sectional estimations [18]. In longitudinal analyses, assessing the effects of adherence to MeDiet on a composite outcome (cardiovascular events incidence, diabetes incidence, or allcause mortality), patients with cancer $(n=809)$, CVD $(n=305)$ and diabetes $(n=355)$ at baseline were excluded. Participants who had not spent enough time in the study to complete and return at least a 2-year follow-up questionnaire $(n=3464)$, and those with missing values for any of the covariates of interest, were also excluded. For the longitudinal analyses, we carefully considered total energy intake and included only plausible reporters (total energy intake/ basal metabolic rate ratio between 1.14 and 2.1) using the cut-off points proposed by Goldberg [19], adjusted for leisure time physical activity. The final effective sample for longitudinal analyses was of 9109 participants. Overall retention rate in the cohort was $91.9 \%$ with a mean follow-up time of 6.2 years (range: $1.6-10.0$ years).

\section{Dietary assessment}

\section{Sample-specific MeDiet score (MDS)}

Dietary habits at baseline were assessed by a 136-item FFQ, validated and described in detail elsewhere $[4,20]$. MDS was calculated as previously described with a 9-point scale $[2,10]$. One point was assigned to persons whose consumption was $\geq$ sex-specific median of components with most likely beneficial effects (vegetables, fruits/nuts, legumes, fish/seafood, cereals, and monounsaturated/saturated [MUFA/SFA] lipid ratio), otherwise no point was assigned; no point was assigned to persons with consumption $\geq$ sexspecific median of components without proven beneficial effects (meat and dairy products), otherwise one point was assigned. For ethanol, one point was assigned to men 
consuming $10-50 \mathrm{~g} /$ day and to women consuming $5-25 \mathrm{~g} /$ day, otherwise, no point was assigned. MDS was subsequently adjusted for total energy intake for each item (residuals method) [21]; in additional analyses, we weighted each MDS items according to the proposed evidence-based contribution to CHD protection [15].

\section{Absolute-normative Mediterranean score (MEDAS) [22]} Adherence to MeDiet was also assessed with the absolutecomponent questionnaire used in the PREDIMED study (MEDAS); 13 items from the original MEDAS (supplementary data) [12-14] were extrapolated using the 136-item FFQ, omitting one of the items (the use of sofrito) because this piece of information could not be extrapolated. A value of 0 or 1 was assigned to each item using the cut-off points that better represented the observed dose-response relationship in previous analyses [12-14]. Then, MEDAS was weighted by the proposed contribution of each component to CHD protection [15]. The elements of MEDAS cannot be adjusted for total energy intake, because they are based on absolute cut-off points.

\section{Other covariates}

Socio-demographic parameters (sex, age), anthropometric measurements (weight, body mass index [BMI]), health related habits (smoking status, physical activity, sedentary lifestyle), and clinical variables (medications, self-reported cardiovascular events, cancer, diabetes, and mortality, which were confirmed by revision of medical records). Selfreported weight and BMI [23], and physical activity assessment [24] were previously validated. Physical activity was expressed in metabolic equivalent tasks (METs = time spent at each activity [hours/week] multiplied by its typical energy expenditure) [25].

\section{Weighting the contribution of MeDiet components}

The usual indexes of adherence to MeDiet assign the same weight to all items regardless of the evidence for each item on cardiovascular prevention. Recent pooled analyses of cohort studies showed that individual dietary components were associated with lower or higher risk of $\mathrm{CHD}$, and the number of Bradford Hill criteria met by each component was different (i.e. 4 criteria for vegetables, 3 for fish, 3 for alcohol, 3 for fruit, 2 for SFA, 3 for MUFA) [15]. We corrected the contribution of MDS and MEDAS items according to the number of Bradford Hill criteria met by each component. Since MDS and MEDAS have a different number of items ( 9 and 13, respectively) we multiplied the original value of each item by the proportion that would achieve the theoretical maximum value of the score and sum the results for a final standardized value of weighted score (example in Supplementary material).

\section{Outcome assessment}

Cardiovascular events were considered for participants reporting acute coronary syndromes, revascularization procedure or stroke on a follow-up questionnaire. An expert panel of physicians, blinded to the information on diet and risk factors, adjudicated the events by reviewing medical records applying the universal criteria for myocardial infarction [26] or clinical criteria for the other outcomes. A non-fatal stroke was defined as a focalneurological deficit of sudden onset and vascular mechanism lasting $>24 \mathrm{~h}$. Probable incident diabetes was considered for participants who reported diabetes diagnosis made by a doctor in a follow-up questionnaire without being diabetic (diagnosis or oral agents/insulin use) at baseline. The diagnosis was then confirmed by reviewing medical records. Details on the date of diagnosis, type of diabetes and other information were requested with additional questionnaires as reported before [4]. Deaths were reported by the person's next kin, work associates and postal authorities. For participants lost to follow-up, the National Death Index was used to identify deceased cohort members. Fatal CHD or stroke were confirmed by a review of medical records with the permission of the next of kin.

\section{Statistical analyses}

We performed the analyses with SPSS software package version 14.0 (SPSS Inc, Chicago, Illinois). Statistical significance was set at two-sided $p<0.05$. We did not use any imputation method for missing data of participants lost to follow-up. Missing data for baseline height $(0.6 \%)$ or weight $(0.2 \%)$ were imputed by using regression models. Tests of linear trend (likelihood ratio test) were conducted across increasing categories of the score by using the score as a continuous variable and adjusting for the potential confounders. Test for heterogeneity (ANCOVA, adjusted for age, sex and baseline BMI) was used to assess differences across scores quintiles. Sex by diet interactions were assessed by including an interaction term in the adjusted models as covariates. Stepwise regression with forward selection was used to evaluate the contribution of each item to the final scores (MDS and MEDAS). Logistic regression models were fit to assess the relationship between baseline adherence to MeDiet (MDS, MEDAS or energyadjusted/weighted versions of these scores) and the incidence of the combined outcome (cardiovascular events incidence, diabetes incidence, or all-cause death). In all analyses, logistic regression models were adjusted for age, sex, BMI, total energy intake, smoking category (never, past and current smokers) and physical activity.

\section{Results}

In addition to be highly educated, the SUN cohort is relatively young (mean age 38.4 years) with predominance of women $(60.5 \%)$, and non-obese participants $(91.3 \%$ with $\mathrm{BMI}<30 \mathrm{~kg} / \mathrm{m}^{2}$ ). Mean physical activity during leisure time was $28.5 \pm 26.3 \mathrm{MET}$-h/week for men and $21.9 \pm 19.1 \mathrm{MET}$ $\mathrm{h} /$ week for women. Socio-demographic characteristics of the studied population according to quintiles of adherence to MDS are shown in Table 1. Most participants had intermediate values of adherence (3-5 points: $62.9 \%$ ) whereas lower ( $\leq 2$ points: $22 \%$ ) or higher values ( $\geq 6$ points: $15.1 \%$ ) were less frequently observed. Participants with higher adherence to MeDiet were older, more likely to be exsmokers, married and more physically active. There was 
Table 1 Characteristics of the study participants according to quintiles of adherence to the Mediterranean diet (MDS, 9-item score). Values are means (range) for age, BMI, and physical activity; percentage for sex, smoking status, and marital status; means for servings.

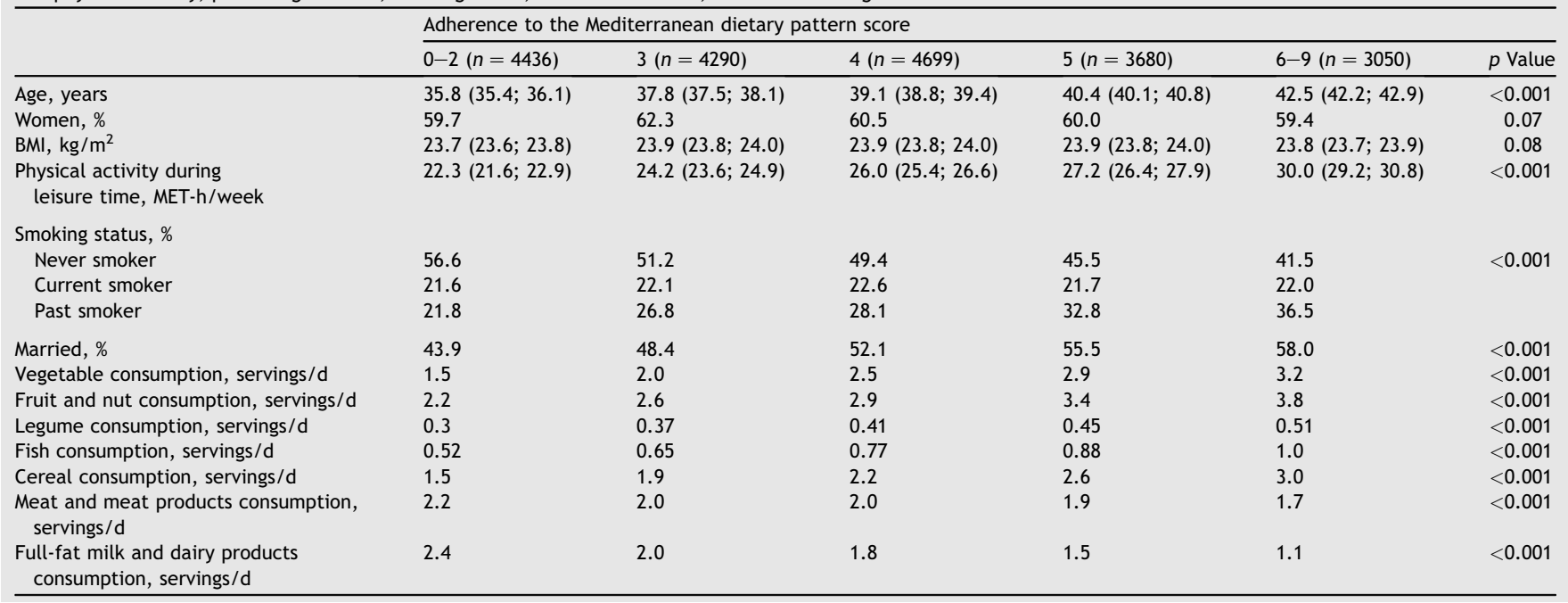


a higher consumption of cereals with increasing values of adherence to MeDiet score, which in the studied population are mostly refined. A stepwise regression analysis with the cumulative percentage of variance of the final score explained by each item of unadjusted MDS and MEDAS is illustrated in Fig. 1. Both scores reflect the diverse components of MeDiet with no single component apparently driving the score; hence, the degree of conformity to MeDiet is the result of the joint impact of all the components of the score and is not strongly influenced by any individual component. Table 2 (Supplementary data) shows the composition (i.e. components with their cutoffs for optimal scoring) for each index.

Table 3 (Supplementary data) shows total energy and macronutrient intake distribution in women and men according to quintiles of adherence to MeDiet, as assessed with MDS and MEDAS. Table 3 also shows the values of MDS after adjusting each item for total energy intake [20] (MDSadj in the table); also shown in the table are values of the scores (MDS [MDS-adj-w] and MEDAS [MEDAS-w]) after both, adjusting the individual items for total energy intake and assigning them differential weights taking into account their evidence-based contribution to CHD protection [15]. Regardless of the score used (MDS, MEDAS, adjusted or not, and weighted or not), both men and women with higher adherence to MeDiet consumed more fiber and alcohol in moderate proportions, and less total fat (especially less saturated fat) with higher MUFA/SFA ratio. The trend of changes for nutrient consumption across different MDS and MEDAS values was similar. This was true also after adjustments and/or differentially weighting the scores. However, total energy intake adjusted with the residual method went

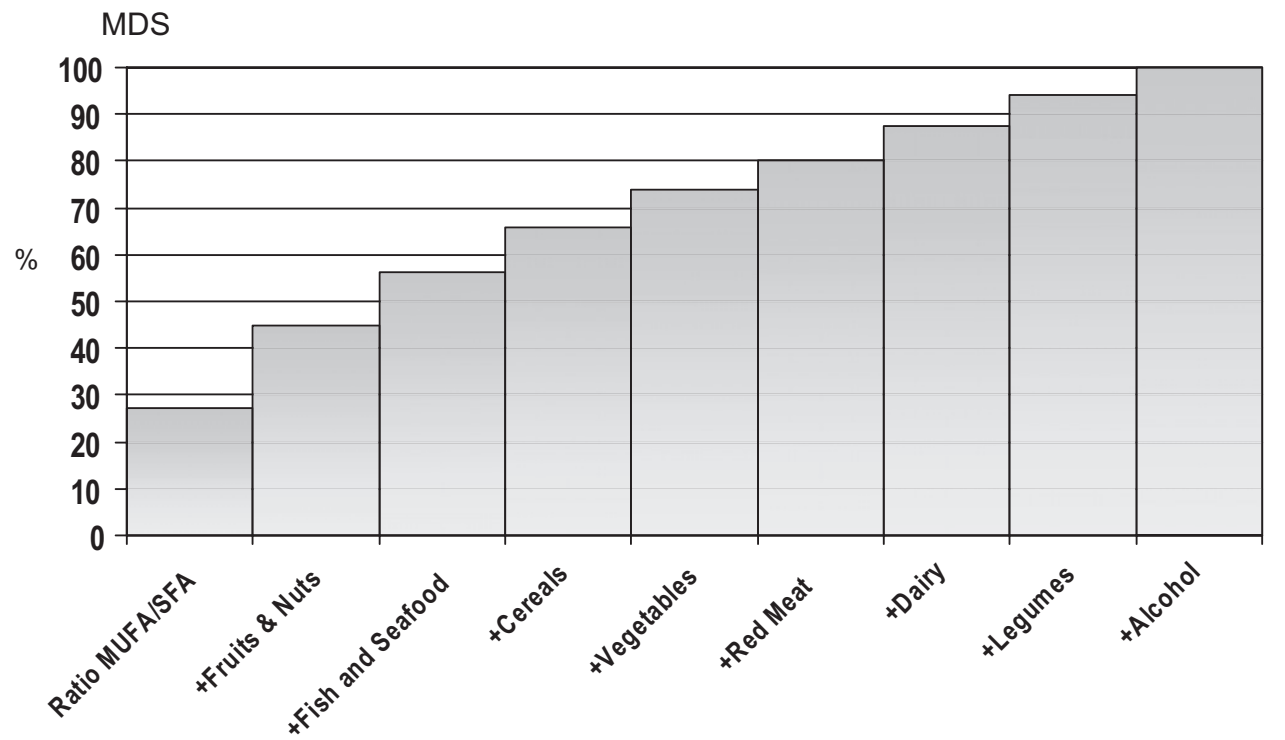

\section{MEDAS}

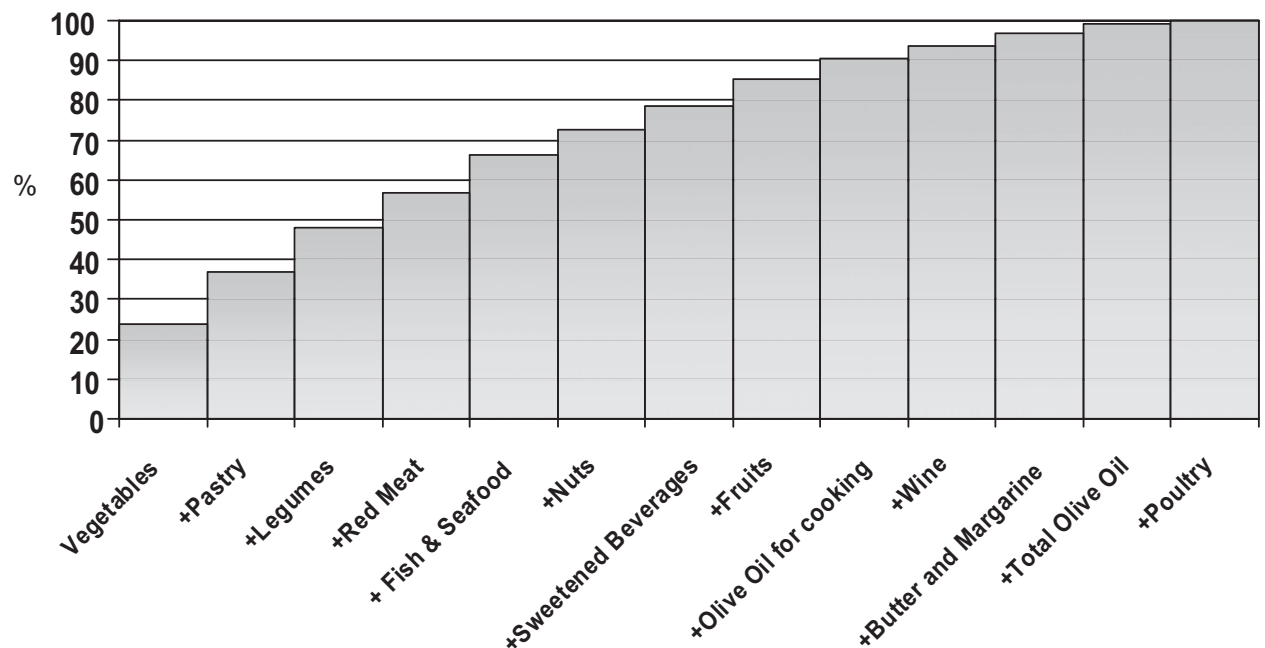

Figure 1 Stepwise regression analysis with the cumulative percentage of variance of the final MeDiet adherence score explained by each item. Upper panel: sample-relative, 9-item Mediterranean diet score (MDS; based on 136-item FFQ). Lower panel: absolute/normative-component Mediterranean diet score (MEDAS; based on 13 questions). 
in an opposite direction when compared to unadjusted values (MDS). Total caloric intake explained 3\% of the MDS variability. But total caloric intake explained only $0.3 \%$ of the MEDAS variability. Adjustment for total energy intake had only a slight effect on subjects' classification. Categorization of subjects in quintiles of adherence according to the MDS changed for only $12 \%$ of participants after adjusting the MDS for total energy intake. This change was even smaller (2.1 and $2.8 \%$, respectively) when the MDS or the MEDAS were weighted according to the strength of the available causal evidence for each item (Table 4, Supplementary material).

As shown in Fig. 2 (Supplementary material), there was a clearly significant increase in adherence to MeDiet with age, regardless of the score used (MDS or MEDAS), which did not change after adjusting or weighting the scores for both men and women.

In the longitudinal analysis, during 92,523 person-year follow-up, there were 58 cases of incident diabetes, 95 incident cases of non-fatal cardiovascular events and 131 deaths. After adjustment for potential confounders (age, sex, BMI, total energy, smoking, physical activity), adherence to MeDiet was associated with a reduced occurrence of the composite outcome (diabetes incidence, cardiovascular events incidence or death) (Table 2), regardless of the score used. As shown in the table, the prediction of the combined outcome was similar for the original scores or for the scores after adjustment for total energy intake or after differentially weighting the specific contribution of each item. When we analyzed the separate components of the composite outcome there were few events for each component to obtain stable estimates of relative risks. This is probably due to the characteristics of the sample with most participants being young. This is an open cohort, hence, future studies may allow us to analyze the effects on single outcomes.

\section{Discussion}

Using data from a large, well-characterized, prospective cohort, we found that a MeDiet score based on 13 questions (absolute-component, MEDAS) was similar to a MeDiet score based on 136-item FFQ (sample-specific, MDS) in categorizing food habits, macronutrient distribution and in its association with a combined outcome of cardiovascular events incidence, diabetes incidence or all-cause mortality. The risk of the combined outcome was significantly lower with a higher adherence to MeDiet, regardless of the score used, even after adjustments for total energy or weighting each item's evidence-based contribution to CHD protection.

The traditional MeDiet has been extensively studied since Keys et al. showed its remarkable association with lower incidence of CVD, cancer, and mortality [27]. Numerous studies have confirmed these and other outcomes $[1-8,10-12,16,28]$, which makes MeDiet the most intensely studied dietary pattern and a paradigm of healthy eating. A recent meta-analysis ( $n>2,000,000$ participants) confirmed these associations and the reduced incidence of various chronic diseases [1], verifying the compelling evidence of protection conferred by adhering to MeDiet. Recently, data from the RCT PREDIMED showed that MeDiet effectively prevents diabetes in subjects at high cardiovascular risk [8], confirming the effects previously reported in observational studies.

The evaluation of food patterns is well-suited with recent attention on the complex interactions among diverse nutrients [29]. The first and most widely used tool to assess adherence to MeDiet, created by Trichopoulou et al. [10] and developed thereafter [2,16], has been extremely useful for epidemiological research, yet, it is not simple to transfer it into everyday clinical practice. The actual amount of each item's consumption in order to adopt MeDiet cannot be established because it is based on sample-specific median consumptions. The data collection (FFQ) to process MDS is time-consuming and there is concern about: i) the inclusion of cereals as a whole irrespectively of glycemic load, fiber content or degree of processing; ii) the difficult quantification of olive oil consumption which has lead to the use of MUFA/SFA, even if some animal products are important sources of MUFA; iii) the inclusion of all sources of alcohol equally. Alternatively, the absolute-normative score (MEDAS) used in the PREDIMED study [12-14] does not use MUFA/SFA but olive oil, is neutral to cereal intake, includes wine, but not other alcohol sources, does not need a FFQ and can be applicable in different settings. This score might facilitate to translate research evidence into practical recommendations for a wide variety of populations. It allows an immediate assessment and feedback to the participants, potentially valuable for nutrition education. The present results

Table 2 Odd ratios (95\% confidence intervals) of a composite outcome (diabetes incidence, cardiovascular event incidence or death) according to adherence to the Mediterranean food pattern (assessed with MDS 9-item score and MEDAS 13-item score), ${ }^{\text {b }}$ before and after weighting the contribution of each item to CHD protection (see text). ${ }^{c}$

\begin{tabular}{|c|c|c|c|c|c|c|}
\hline & $\begin{array}{l}\text { MDS }(9 \text {-item) } \\
\text { per } 2 \text { points }\end{array}$ & $\begin{array}{l}p \text { for } \\
\text { trend }\end{array}$ & $\begin{array}{l}\text { MDS-adj }{ }^{\mathrm{a}} \\
\text { per } 2 \text { points }\end{array}$ & $\begin{array}{l}p \text { for } \\
\text { trend }\end{array}$ & $\begin{array}{l}\text { MEDAS (13-item) } \\
\text { per } 3 \text { points }\end{array}$ & $\begin{array}{l}p \text { for } \\
\text { trend }\end{array}$ \\
\hline Not weighted & $0.80(0.66-0.98)$ & 0.03 & $0.79(0.66-0.94)$ & 0.01 & $0.76(0.57-0.99)$ & 0.03 \\
\hline Weighted & $0.81(0.67-0.97)$ & 0.02 & $0.81(0.69-0.95)$ & $<0.01$ & $0.75(0.58-0.97)$ & 0.03 \\
\hline
\end{tabular}

MDS: Mediterranean diet score (9-item); MEDAS: absolute/normative Mediterranean diet score (13 item).

${ }^{a}$ MDS-adj: MDS adjusted for total energy intake (residual method).

b The elements used to build the MEDAS score cannot be individually adjusted for total energy intake, because they are based on absolute cut-off points.

${ }^{c}$ In all analyses, logistic regression models were adjusted for age, sex, BMI, total energy intake, smoking category (never, past and current smokers) and physical activity. 
demonstrate similar prediction of macronutrient composition and crucial health outcomes with MEDAS and MDS, even after adjustments, confirming their comparability.

Adherence to MeDiet increased with age regardless of the score used. Older participants may have less likely abandoned the traditional food pattern versus the appeal for Westernized food in younger participants, as reported in Mediterranean countries [30]. Younger populations may not be aware of the long-term drawbacks of an unhealthy diet, which should be considered when planning prevention strategies.

The present study has a number of strengths, including a large sample size, high retention rate, prospective design, and lengthy follow-up. Potential limitations include self-reported information. However, parameters such as self-reported weight/BMl have been previously validated [23], hence, possible residual confounding due to errors in BMI and physical activity should be minimal. The cohort, composed mostly of middle-aged, Spanish educated subjects, may make generalizability of results not practical, although it reduces confounding by disease, education, socioeconomic status, and presumed access to health care. Future studies in other populations are warranted to test the applicability of MEDAS. We used a validated FFQ [20], which means that possible recall bias is the same for future cases and non-cases. We acknowledge that the use of Bradford Hill criteria is currently a matter of debate since meeting these criteria is not the same as defining the strength of an association (e.g., the effect of vegetables may be most plausible based on these criteria, but may have per unit a smaller effect on the CHD risk as compared to, for example, SFA/PUFA). In the present study the results were not significantly different after weighing the scores with these criteria.

In conclusion, after a median follow-up of 6.2 years, a lower occurrence of a combined outcome including diabetes incidence, cardiovascular events incidence or total mortality was associated with increased adherence to MeDiet. The association was similar if based on a 13question score with absolute-normative cut-off points or on a 136-FFQ sample-specific medians of consumption as cut-off points. Prediction of macronutrient distribution was also similar for both scores. Time has come to translate compelling positive evidence of MeDiet into practical applications.

\section{Role of the sponsors}

Funding sources had no role in the design and conduct of the study; collection, management, analysis, and interpretation of the data; and preparation, review, or approval of the manuscript.

\section{Funding/support}

The SUN Study has received funding from the Spanish Ministry of Health (Grants PI01/0619, PI030678, PI040233, PI042241, PI050976, PI070240, PI070312, PI081943, PI080819, RD06/0045, and G03/140), the Navarra Regional Government (36/2001, 43/2002, 41/2005, 36/2008) and the University of Navarra.

\section{Conflict of interest}

There were no conflicts of interest.

\section{Acknowledgments}

The authors thank the participants for their enthusiastic collaboration and acknowledge the collaboration of the SUN Project study group: Alonso A, Basterra-Gortari J, Benito S, de Irala J, Delgado-Rodriguez M, Guillen-Grima F, Krafka J, Llorca J, Lopez del Burgo C, Marti A, Martinez JA, Nuñez-Cordoba JM, Pimenta AM, Sanchez D, SanchezVillegas A, Segui-Gomez M, Serrano-Martínez M, and Vazquez $\mathrm{Z}$.

\section{Appendix. Supplementary data}

Supplementary data associated with this article can be found, in the online version, at doi:10.1016/j.numecd. 2011.10.009.

\section{References}

[1] Sofi F, Abbate R, Gensini GF, Casini A. Accruing evidence about benefits of adherence to the Mediterranean diet on health: an updated systematic review and meta-analysis. Am J Clin Nutr 2010;92:1189-96.

[2] Trichopoulou A, Costacou T, Bamia C, Trichopoulos D. Adherence to a Mediterranean diet and survival in a Greek population. N Engl J Med 2003;348:2599-608.

[3] Mitrou PN, Kipnis V, Thiebaut AC, Thiébaut ACM, Reedy J, Subar AF, et al. Mediterranean dietary pattern and prediction of all-cause mortality in a US population: results from the $\mathrm{NIH}$ AARP diet and health study. Arch Intern Med 2007;167: 2461-8.

[4] Martinez-Gonzalez MA, de la Fuente-Arrillaga C, NunezCordoba JM, Basterra-Gortari FJ, Beunza JJ, Vazquez Z, et al. Adherence to Mediterranean diet and risk of developing diabetes: prospective cohort study. BMJ 2008;336: 1348-51.

[5] Fung TT, Rexrode KM, Mantzoros CS, Manson JE, Willett WC, $\mathrm{Hu}$ FB. Mediterranean diet and incidence of and mortality from coronary heart disease and stroke in women. Circulation 2009;119:1093-100.

[6] Martinez-Gonzalez MA, Garcia-Lopez M, Bes-Rastrollo M, Toledo E, Martínez-Lapiscina EH, Delgado-Rodriguez M, et al. Mediterranean diet and the incidence of cardiovascular disease: a Spanish cohort. Nutr Metab Cardiovasc Dis; 2010. Jan 20. [Epub ahead of print].

[7] Sanchez-Villegas A, Delgado-Rodriguez $M$, Alonso A, Schlatter J, Lahortiga F, Serra Majem L, et al. Association of the Mediterranean dietary pattern with the incidence of depression: the Seguimiento Universidad de Navarra/University of Navarra follow-up (SUN) cohort. Arch Gen Psychiatry 2009;66:1090-8.

[8] Salas-Salvadó J, Bulló M, Babio N, Martínez-González MÁ, Ibarrola-Jurado N, Basora J, et al. Reduction in the incidence of type 2 diabetes with the Mediterranean diet. Results of the PREDIMED-Reus nutrition intervention randomized trial. Diabetes Care 2011;34:14-9.

[9] Bach A, Serra-Majem L, Carrasco JL, Roman B, Ngo J, Bertomeu I, et al. The use of indexes evaluating the 
adherence to the Mediterranean diet in epidemiological studies: a review. Public Health Nutr 2006;9:132-46.

[10] Trichopoulou A, Kouris-Blazos A, Wahlqvist ML, Gnardellis C, Lagiou P, Polychronopoulos E, et al. Diet and overall survival in elderly people. BMJ 1995;311:1457-60.

[11] Kouris-Blazos A, Gnardellis C, Wahlqvist ML, Trichopoulos D, Lukito W, Trichopoulou A. Are the advantages of the Mediterranean diet transferable to other populations? A cohort study in Melbourne, Australia. Br J Nutr 1999;82:57-61.

[12] Estruch R, Martinez-Gonzalez MA, Corella D, Salas-Salvadó J, Ruiz-Gutiérrez V, Covas MI, et al. Effects of a Mediterraneanstyle diet on cardiovascular risk factors: a randomized trial. Ann Intern Med 2006;145:1-11.

[13] Shröder H, Fitó M, Estruch R, Martínez-González MA, Corella D, Salas-Salvadó J, et al. A short screener is valid for assessing Mediterranean diet adherence among older Spanish men and women. J Nutr 2011;141:1140-5.

[14] Martínez-González MA, Corella D, Salas-Salvadó J, Ros E, Covas MI, Fiol M, et al. Cohort Profile: design and methods of the PREDIMED study. Int J Epidemiol; 2010. Dec 20. [Epub ahead of print] PubMed PMID: 21172932.

[15] Mente A, de Koning L, Shannon HS, Anand SS. A systematic review of the evidence supporting a causal link between dietary factors and coronary heart disease. Arch Intern Med 2009;169:659-69.

[16] Trichopoulou A, Bamia C, Trichopoulos D. Anatomy of health effects of Mediterranean diet: Greek EPIC prospective cohort study. BMJ 2009;338:b2337.

[17] Segui-Gomez M, de la Fuente C, Vazquez Z, de Irala J, Martinez-Gonzalez MA. Cohort profile: the 'Seguimiento Universidad de Navarra' (SUN) study. Int J Epidemiol 2006;35: 1417-22.

[18] Willett W. Nutritional epidemiology. 2nd ed. New York: Oxford University Press; 1998.

[19] Goldberg GR, Black AE, Jebb SA, Cole TJ, Murgatroyd PR, Coward WA, et al. Critical evaluation of energy intake data using fundamental principles of energy physiology: 1 . Derivation of cut-off limits to identify under-recording. Eur J Clin Nutr 1991;45:569-81.

[20] de la Fuente-Arrillaga C, Vazquez Ruiz Z, Bes-Rastrollo $M$, Sampson L, Martinez-Gonzalez MA. Reproducibility of an FFQ validated in Spain. Public Health Nutr 2010;13:1364-72.
[21] Hu FB, Stampfer MJ, Rimm E, Ascherio A, Rosner BA, Spiegelman D, et al. Dietary fat and coronary heart disease: a comparison of approaches for adjusting for total energy intake and modeling repeated dietary measurements. Am J Epidemiol 1999;149:531-40.

[22] Martinez-Gonzalez MA, Fernandez-Jarne E, SerranoMartinez M, Wright M, Gomez-Gracia E. Development of a short dietary intake questionnaire for the quantitative estimation of adherence to a cardioprotective Mediterranean diet. Eur J Clin Nutr 2004;58:1550-2.

[23] Bes-Rastrollo M, Perez Valdivieso JR, Sanchez-Villegas A, Alonso A, Martinez-Gonzalez MA. Validación del peso e índice de masa corporal auto-declarados de los participantes de una cohorte de graduados universitarios. Rev Esp Obes 2005;3: 183-9.

[24] Martinez-Gonzalez MA, Lopez-Fontana C, Varo JJ, SanchezVillegas A, Martinez JA. Validation of the Spanish version of the physical activity questionnaire used in the nurses' health study and the health professionals' follow-up study. Public Health Nutr 2005;8:920-7.

[25] Ainsworth BE, Haskell WL, Whitt MC, Irwin ML, Swartz AM, Strath SJ, et al. Compendium of physical activities: an update of activity codes and MET intensities. Med Sci Sports Exerc 2000;32:5498-504.

[26] Thygesen K, Alpert JS, White HD, Jaffe AS, Apple FS, Galvani $M$, et al. Universal definition of myocardial infarction. Circulation 2007;116:2634-53.

[27] Keys A, Menotti A, Karvonen MJ, Aravanis C, Blackburn H, Buzina R, et al. The diet and 15-year death rate in the seven countries study. Am J Epidemiol 1986;124:903-15.

[28] Knoops KT, de Groot LC, Kromhout D, Perrin AE, MoreirasVarela $\mathrm{O}$, Menotti A, et al. Mediterranean diet, lifestyle factors, and 10-year mortality in elderly European men and women: the HALE project. JAMA 2004;292:1433-9.

[29] Jacobs Jr DR, Gross MD, Tapsell LC. Food synergy: an operational concept for understanding nutrition. Am J Clin Nutr 2009;89:1543S-8S.

[30] Sanchez-Villegas A, Delgado-Rodriguez M, MartinezGonzalez MA, De Irala-Estevez J. Gender, age, sociodemographic and lifestyle factors associated with major dietary patterns in the Spanish project SUN (Seguimiento Universidad de Navarra). Eur J Clin Nutr 2003;57:285-92. 